ISSN: 2304-0335 DOI: https://doi.org/10.17162/au.v10i1.418

\title{
Eficacia del programa ¡Qué importante es tributar! en el desarrollo de cultura tributaria en adolescentes
}

\section{Effectiveness of the program "How important it is to pay taxes!" in the development of tax education in teenagers}

\author{
Samuel Condori Loayza ${ }^{1}$, Oscar Mamani-Benito ${ }^{2 \mathrm{a}} \mathrm{y}$ David Hugo Bernedo Moreira ${ }^{3}$ \\ Universidad Peruana Unión, Juliaca, Perú ${ }^{12}$ \\ Instituto de Altos Estudios en Investigación y Desarrollo Empresarial, Puno, Perú ${ }^{3}$
}

Orcid ID: https://orcid.org/0000-0002-0759-8645 1

Orcid ID: https://orcid.org/0000-0002-9818-26012

Orcid ID: https://orcid.org/0000-0002-0759-8645 3

Recibido: 05 de junio de 2019

Aceptado: 12 de octubre de 2019

\begin{abstract}
Resumen
El presente estudio tuvo el objetivo de determinar la eficacia del programa educativo ¡Qué importante es tributar! orientado a desarrollar conocimientos y actitudes favorables sobre cultura tributaria en estudiantes de secundaria de una institución educativa privada de Juliaca. La investigación se enmarca en un diseño cuasi-experimental con evaluación pre y post intervención, en el cual los participantes fueron distribuidos en dos grupos: experimental (GE $=30)$ y control $(\mathrm{GC}=30)$. En este caso, fue el GE quien recibió un total de 10 sesiones educativas distribuidas en 3 módulos: conocimientos, percepción y actitudes. Para determinar la eficacia del programa se aplicó un cuestionario que medía la cultura tributaria utilizada en una investigación similar y validada por expertos en educación financiera. Los resultados indican que antes de la intervención seis participantes del GE (20\%) demostraron conocimientos sobre cultura tributaria a nivel bajo, 19 a nivel moderado $(63.3 \%)$ y cinco a nivel alto (16.7\%); luego de la intervención, ninguno demostró un nivel bajo, 19 un nivel moderado $(63.3 \%)$ y 11 un nivel alto. Según la prueba de hipótesis, el estadístico $t$ de student reveló diferencias significativas entre los puntajes hallados antes y después de la intervención $(\mathrm{t}=-$ $7.509, \mathrm{p}<.05)$, mientras que las diferencias en el grupo control fueron porcentuales mas no significativas $(\mathrm{t}=.648, \mathrm{p}>.05)$. Se concluye que el programa educativo incrementó positivamente conocimientos, percepciones y actitudes favorables hacia la cultura tributaria en adolescentes, a fin de promover el cumplimiento ético de las obligaciones tributarias.
\end{abstract}

aCorrespondencia al autor

E-mail: oscar.mb@upeu.edu.pe 
Palabras clave: Cultura tributaria; evasión tributaria; educación financiera, economía

\begin{abstract}
The present study aimed to determine the effectiveness of the educational program "How important it is to pay taxes!" oriented to develop knowledge and favorable attitudes about tax culture in high school students of a private educational institution of Juliaca. The research is framed in a quasi-experimental design with pre and post intervention evaluation, in which the participants were divided into two groups: experimental $(\mathrm{GE}=30)$ and control $(\mathrm{GC}=30)$. In this case, it was the GE who received a total of 10 educational sessions distributed in 3 modules: knowledge, perception and attitudes. To determine the effectiveness of the program, a questionnaire was applied that measured the tax culture used in a similar investigation and validated by experts in financial education. The results indicate that before the intervention six participants of the GE (20\%) demonstrated knowledge about tax culture at a low level, 19 at a moderate level (63.3\%) and five at a high level (16.7\%); after the intervention, none showed a low level, 19 a moderate level (63.3\%) and 11 a high level. According to the hypothesis test, the student $t$ statistic revealed significant differences between the scores found before and after the intervention $(\mathrm{t}=-7.509, \mathrm{p}<.05)$, while the differences in the control group were more nonsignificant percentages $(t=.648, p>.05)$. It is concluded that the educational program positively increased knowledge, perceptions and favorable attitudes towards the tax culture in adolescents, in order to promote ethical compliance with tax obligations.
\end{abstract}

Keywords: Tax Culture; Tax Evasion; Financial Education, Economics

\title{
Introducción
}

Entre el ciudadano y el estado existe una relación de acentuada tenacidad, producto del cambio de las políticas fiscales y del ciclo económico, las cuales podrían resultar en un saldo positivo o negativo. Dicho de otra forma, y en concordancia con Camarero, Del Pino y Mañas (2015), si el contribuyente percibe que el empoce de impuestos que realiza al estado no es proporcional a lo que recibe, entonces este no compensa las aportaciones, y por ende, surge un saldo negativo; de allí nace la idea de una cultura tributaria, es decir, se valida el punto de vista del ciudadano para tributar voluntariamente, y se establece como norma colectiva implícita, dándole sentido a la función redistribuidora del mismo estado.

En nuestro país, el sistema tributario es tan complejo que desmotiva su formalización, convirtiéndose en una de las principales razones para la conducta de evasión. Al igual que algunos países en Latinoamérica (Zamora, 2018; Gómez \& Morán, 2016) en el Perú existe una problemática latente respecto a la evasión tributaria y su incidencia en la economía nacional (López, 2014; Lahura, 2016); no obstante, se ha observado en los últimos años un crecimiento y progreso, el cual se ha sostenido gracias a factores externos-como el alza de los metales y 
otras materias primas en el mercado internacional (INEI, 2018). Sin embargo, la sostenibilidad y la satisfacción de las necesidades públicas no pueden depender de un ingreso tan volátil, y por ello, el estado busca reducir la brecha fiscal representada en un 36\% de evasión de impuesto general a las ventas (IGV) y 51\% del Impuesto a la Renta (IR) (Oliva, 2018), a fin de asegurar la satisfacción de las necesidades sociales.

En opinión de Estrada (2018), la Superintendencia de Aduanas y Administración Tributaria (SUNAT) — como entidad recaudadora—busca propiciar al estado los recursos necesarios para su administración, ejerciendo constante presión sobre la población de contribuyentes ya existentes y colocando sobre ellos una gran carga impositiva, la cual casi pone en riesgo la continuidad empresarial de los mismos. Esta presión podría aliviarse si el estado se enfocara en buscar la ampliación de la base tributaria, es decir, incrementar el número de contribuyentes en lugar de presionar a los ya existentes.

Por ello, a fin de cambiar el rumbo de la situación fiscal en el Perú, se hace indispensable una herramienta trascendental en la aplicación de estrategias dirigidas a niños y adolescentes tratando de concientizar la responsabilidad social de los ciudadanos. Algunos de estos programas fueron denominados como "Educación Fiscal," "Educación tributaria" o "Cultura Tributaria" (Zambrano, 2019). Precisamente Alcos (2016), mediante una investigación respecto a la cultura tributaria en el nivel de educación básica regular de la región Puno, evidenció la falta de conocimientos suficientes en materia tributaria en niños, jóvenes y docentes del nivel primario y secundario, ya que el currículo de enseñanza no se adapta para educar tributariamente y mucho menos generar conciencia tributaria. Por otra parte, Mostacero y Vásquez (2014) realizaron un análisis comparativo de la aplicación de un programa de orientación y cultura tributaria en estudiantes del quinto año de secundaria de instituciones educativas públicas y privadas de la ciudad de Trujillo, que al impartir conocimientos de formación sustancial y aplicando un programa de inducción a la conciencia tributaria-social, los estudiantes mejoraron su cultura tributaria. Con esto quedó demostrado que este tipo de estrategias de formación permite el impulso de que los contribuyentes se muestren responsables y que cumplan con su deber hacia el estado.

En consecuencia, partiendo de la premisa de Ruiz (2017), quien refiere que es necesario introducir componentes pedagógicos para promover la cultura tributaria — vinculando el sentido de pertenencia con el sistema tributario, ampliando la conciencia de deberes y derechos, la corrección de la evasión y la elusión, el respeto y uso racional de los bienes y servicios públicos-el objetivo del presente estudio es determinar si el programa educativo "iQue 
importante es tributar!" es eficaz en el desarrollo de una cultura tributaria en adolescentes en etapa de formación educativa.

\section{Método}

\section{Diseño}

El presente trabajo exhibe un estudio cuasi-experimental con grupo control, mediante la evaluación pre y post intervención. Esto se elabora a fin de contrastar la exposición, respuesta e hipótesis de la investigación, y debido a la carencia de aleatorización de los sujetos a los grupos de tratamiento y control (Nass \& Merino, 2008).

\section{Participantes}

La población de estudio estuvo conformada por dos grupos: experimental y control. Debido al diseño planteado en esta investigación, se trabajó con muestras o grupos intactos. En tal sentido, el grupo experimental estuvo conformado por 30 participantes de ambos sexos pertenecientes al quinto año de secundaria de una institución educativa de la ciudad de Juliaca; de igual forma, el grupo control estuvo conformado por 30 participantes de ambos sexos, de la misma institución y el mismo grado, pero de diferente sección.

\section{Instrumentos}

A fin de determinar la eficacia del programa se utilizó un cuestionario para medir la cultura tributaria de los participantes, el cual fue elaborado por Vera (2017) en la ciudad de Lima y validada para efectos de la presente investigación por tres docentes universitarios especialistas en educación tributos y educación financiera. Este instrumento consta de 20 preguntas con opciones de respuesta múltiple ( $1=$ si, $2=$ no, $3=$ no sé de lo que se habla), que a su vez evalúa tres dimensiones: "conocimientos de la naturaleza social de la tributación," "percepción respecto al deber de tributar" y "actitud frente a la obligación de tributar." Para determinar el valor de sus propiedades psicométricas se procedió a validar el contenido mediante el juicio de tres expertos, colegiados y especialistas en tributación. La valoración fue promediada en un índice de acuerdo que oscila entre 0 a 1 , siendo el corte .85 indicador de adecuada validez de contenido; en este caso, el valor obtenido fue I/A = .95; aunado a esto, el coeficiente de confiabilidad Alpha de Cronbach demostró un valor de $=.89$; por lo tanto, se asume que el instrumento es válido y altamente confiable.

\section{Procedimientos}


El estudio se ejecutó en tres fases: pre-test, intervención y post-test. Una vez obtenidos los indicadores de validez y la confiabilidad del instrumento, se procedió a coordinar la primera aplicación (pre-test), tanto en el grupo control como en el experimental. Para ello, se coordinó con las autoridades de la institución una fecha en la que los participantes de ambos grupos dispongan de 30 minutos para desarrollar la encuesta. Una semana después de la primera aplicación se inició la fase de aplicación del programa de intervención solo al grupo experimental.

En cuanto al programa "¿Qué importante es tributar!," este fue elaborado por el autor principal del estudio, quien configuró las sesiones educativas con insumos de las guías pedagógicas que se encuentran disponibles en la página web de SUNAT (2019). El programa consta de tres módulos: (1) conocimiento, que implica el desarrollo de cultura cívica, derechos y responsabilidades y convivencia democrática; (2) percepción, que implica el desarrollo de participación ciudadana, administración del estado, transparencia y corrupción; y (3) actitud, en el que se desarrolló temáticas como conducta adversa de colaboración, incumplimiento formal e informalidad comercial. La aplicación tuvo una duración de dos meses (octubre a noviembre de 2018), se desarrollaron 10 sesiones de entre 30 a 45 minutos de duración, utilizando una metodología participativa con talleres vivenciales.

Finalmente, culminada la fase de intervención, se procedió a ejecutar la fase del posttest. Para ello, se manejó el mismo instrumento utilizado en la primera evaluación. Cabe resaltar que, en esta instancia, se aplicó el instrumento a ambos grupos, tanto el experimental como el de control.

\section{Consideraciones éticas}

Antes de la ejecución del programa se procedió a solicitar la autorización por parte de la institución involucrada, la misma que fue otorgada por el comité de ética a cargo de la administración educativa. Asimismo, a cada participante le fue explicado las implicancias de su participación antes de ejecutar el pre test, poniendo énfasis en el hecho de que la información personal brindada en el cuestionario demográfico iba a tener un manejo totalmente confidencial, asignándose solo códigos para la identificación de cada participante. Una vez obtenido su consentimiento informado y el de sus padres se procedió a ejecutar el programa.

\section{Análisis de datos}

Configurada la matriz en Excel-las mismas que contenían las respuestas del cuestionario-se procedió al análisis en el software SPSS versión 22.0, generando tablas de 
frecuencia y porcentaje para el análisis descriptivo y sociodemográfico. Así también, para el análisis inferencial se aplicó la prueba de normalidad, donde el test Shapiro-Wilk orientó el uso de estadísticos paramétricos para la prueba de hipótesis.

\section{Resultados}

En la tabla 1 se observa las características demográficas correspondientes al grupo experimental y control. En caso del grupo experimental, el 53.3\% de participantes fueron varones y el $46.7 \%$ mujeres; la edad predominante fue de 16 años (80\%); el 90\% proviene de provincias y distritos de la sierra y el $90 \%$ se considera de nivel socioeconómico medio. En caso de sus progenitores, $46.7 \%$ (los padres) y $63.3 \%$ (las madres) culminaron la secundaria; no obstante, $60 \%$ (de padres) y $80 \%$ (de madres) se ocupan como trabajadores independientes.

En la misma tabla, en caso del grupo control, la edad con mayor predominancia fue de 16 años (80\%); el 53.3\% de participantes fueron varones y el $46.7 \%$ mujeres; el $96.7 \%$ proviene de provincias y distritos de la sierra y un $90 \%$ se considera de un nivel socioeconómico medio. En caso de sus progenitores, $53.3 \%$ (de padres) y $63.3 \%$ (de madres) alcanzaron un nivel de instrucción correspondiente a secundaria completa. No obstante, $67.7 \%$ (padres) y $76.7 \%$ (madres) se ocupan como trabajadores independientes.

Tabla 1

Análisis sociodemográfico de los grupos de investigación experimental y control

\begin{tabular}{|c|c|c|c|c|c|}
\hline \multirow[t]{2}{*}{ Variables } & & \multicolumn{2}{|c|}{ Grupo experimental } & \multicolumn{2}{|c|}{ Grupo control } \\
\hline & & $\mathrm{f}$ & $\%$ & $\mathrm{f}$ & $\%$ \\
\hline \multirow[t]{4}{*}{ Edad } & 15 años & 2 & 6.7 & 1 & 3.3 \\
\hline & 16 años & 24 & 80.0 & 24 & 80.0 \\
\hline & 17 años & 3 & 10.0 & 4 & 13.3 \\
\hline & 18 años & 1 & 3.3 & 1 & 3.3 \\
\hline \multirow[t]{2}{*}{ Sexo } & Masculino & 16 & 53.3 & 16 & 53.3 \\
\hline & Femenino & 14 & 46.7 & 14 & 46.7 \\
\hline \multirow[t]{2}{*}{ Procedencia } & Costa & 3 & 10.0 & 1 & 3.3 \\
\hline & Sierra & 27 & 90.0 & 29 & 96.7 \\
\hline \multirow[t]{3}{*}{ Nivel socioeconómico } & Alta & 2 & 6.7 & 0 & 0 \\
\hline & Media & 27 & 90.0 & 27 & 90.0 \\
\hline & Baja & 1 & 3.3 & 3 & 10.0 \\
\hline \multirow[t]{3}{*}{ Nivel de instrucción del padre } & Primaria & 6 & 20.0 & 3 & 10.0 \\
\hline & Secundaria & 14 & 46.7 & 16 & 53.3 \\
\hline & Superior & 10 & 33.3 & 11 & 36.7 \\
\hline \multirow[t]{2}{*}{ Nivel de instrucción de la madre } & Primaria & 6 & 20.0 & 4 & 13.3 \\
\hline & Secundaria & 19 & 63.3 & 19 & 63.3 \\
\hline
\end{tabular}




\begin{tabular}{llcrrr}
\hline & Superior & 5 & 16.7 & 7 & 23.3 \\
Ocupación del padre & Profesional & 12 & 40.0 & 10 & 33.3 \\
& No profesional & 18 & 60.0 & 20 & 66.7 \\
Ocupación de la madre & Profesional & 6 & 20.0 & 7 & 23.3 \\
& No profesional & 24 & 80.0 & 23 & 76.7 \\
\hline
\end{tabular}

Fuente: Elaboración propia.

Por otro lado, en la tabla 2 el grupo experimental—antes de aplicar el programa de intervención-el $20 \%$ de participantes tenía un nivel bajo de conocimientos sobre cultura tributaria, un $63.3 \%$ un nivel moderado y un 16.7 un nivel alto; luego de la intervención, no se evidenció algún participante con nivel bajo de conocimientos, 63.3\% mantenía el nivel moderado y $36.7 \%$ demostraba un nivel alto.

En la misma tabla 2, en el grupo control se identificó que $20 \%$ de los participantes demostraron un nivel bajo de conocimientos sobre cultura tributaria, 63.3\% un nivel moderado y $16.7 \%$ un nivel alto, producto del pre-test. Posteriormente, como producto del post-test se identificó que aún el 20\% demostraba un nivel bajo de conocimientos, $76.7 \%$ un nivel moderado y $16.7 \%$ un nivel alto.

Tabla 2

Nivel de conocimientos expresado en frecuencias antes y después de la intervención

\begin{tabular}{llcccccccc}
\hline & \multicolumn{9}{c}{ Nivel de cultura tributaria } \\
\cline { 3 - 11 } & & \multicolumn{3}{c}{ Bajo } & \multicolumn{2}{c}{ Moderado } & \multicolumn{2}{c}{ Alto } & \multicolumn{2}{c}{ Total } \\
& & F & $\%$ & f & $\%$ & f & $\%$ & f & $\%$ \\
\hline \multirow{3}{*}{ Grupo experimental } & Pre test & 6 & 20.0 & 19 & 63.3 & 5 & 16.7 & 30 & 100 \\
& Post test & 0 & 0.0 & 19 & 63.3 & 11 & 36.7 & 30 & 100 \\
& Pre test & 6 & 20.0 & 23 & 76.7 & 1 & 3.3 & 30 & 100 \\
& Post test & 6 & 20.0 & 19 & 63.3 & 5 & 16.7 & 30 & 100 \\
\hline
\end{tabular}

Fuente: Elaboración propia.

En cuanto al análisis inferencial, tal como se ha revelado en la tabla 2, las diferencias porcentuales obtenidas luego de la aplicación del programa son más notorias en el grupo experimental que en el grupo control. Esto también es corroborado en la tabla 3, donde se observa que, en el grupo experimental, el puntaje de la media en el post-test (33.16) es mayor al puntaje obtenido en el pre-test (30.73), a diferencia del grupo control, donde se observa que el puntaje de la media en el post-test (30.83) es ligeramente mayor que el puntaje de la media en el pre-test (30.23). Según la prueba de hipótesis-la cual involucró un estadístico 
paramétrico en base a los valores obtenidos por Shapiro-Wilk (p > .05)-las diferencias encontradas son significativas en el grupo experimental $(t=-7.509, \mathrm{p}<.05)$, mas no en el grupo control $(\mathrm{t}=-.648, \mathrm{p}>.05)$.

Tabla 3

Estadísticos de grupo para la prueba de hipótesis

\begin{tabular}{llccccc}
\hline & & Media & $\begin{array}{c}\text { Desviación } \\
\text { estándar }\end{array}$ & $\begin{array}{c}\text { Shapiro } \\
\text { Wilk }\end{array}$ & $\mathrm{t}$ & $\mathrm{p}$ \\
\hline Experimental & Pre-test & 30,7333 & 3,41329 & .656 & -7.509 & .000 \\
& Post-test & 33,1667 & 3,00670 & .138 & & \\
Control & Pre-test & 30,2333 & 3,25559 & .067 & -.648 & .522 \\
& Post-test & 30,8333 & 3,76997 & .930 & & \\
\hline
\end{tabular}

Fuente: Elaboración propia.

\section{Discusión}

Los resultados de la investigación orientan a comprender la importancia de educar en cultura tributaria en etapas de formación educativa para los adolescentes. Precisamente, uno de los hallazgos principales recae sobre el hecho de que el programa "iQué importante es tributar!", orientado a desarrollar cultura tributaria, fue eficaz en la concientización del cumplimiento voluntario de los deberes tributarios dentro del contexto de la normatividad que la rige (Ruiz, 2017). Así lo respaldan los resultados obtenidos con el estadístico de prueba, que indican que las diferencias en las puntuaciones antes y después de la intervención en el grupo experimental fueron significativas $(\mathrm{t}=-7.509, \mathrm{p}<.05)$. En cambio, en el grupo control一aunque hubo diferencias porcentuales_estas no demuestran significancia alguna $(\mathrm{t}=-.648, \mathrm{p}>.05) . \mathrm{Al}$ respecto, una explicación del incremento porcentual (de 1 a 5 participantes) con nivel alto de conocimientos sobre cultura tributaria en el grupo control, puede surgir a raíz de las condiciones educativas de este grupo; por una parte, los medios de comunicación y la coyuntura de noticias sobre evasión tributaria y problemas judiciales en la región Puno (además de la logística aplicada en el grupo experimental—sesiones educativas con contenido temático llamativo) pudieron haber despertado el interés de los participantes del grupo control quienes podrían haber generado algún aprendizaje sobre el tema.

Los hallazgos positivos en la presente investigación concuerdan con lo encontrado por Mostacero y Vasquez (2014), quienes realizaron una investigación similar en Trujillo, que tuvo el objetivo de aplicar un programa de orientación y cultura tributaria en estudiantes del quinto año de nivel secundario en instituciones privadas y públicas. Sus resultados revelan que el 
incremento del nivel de conciencia respecto al cumplimiento tributario y el desarrollo de una sensación de riesgo efectivo por el debido incumplimiento. A diferencia del presente estudio, se trabajó con una población de 120 alumnos, teniendo dos grupos de carácter experimental y dos de control; sin embargo, este aspecto no resta validez a los resultados encontrados en la presente investigación, pues solo se diferencia en la capacidad de generalización mas no en la efectividad de programas de naturaleza educativa en el ámbito tributario. Aunado a esto, una explicación teórica que justifica la eficacia de este tipo de propuestas de intervención puede ser encontrada en lo mencionado por Sanchez, Sartori y Salluca (2017). Estos desarrollaron una investigación orientada a determinar la influencia de la educación tributaria y demostrando que, impartiendo lecciones de tributación a ciudadanos en etapa escolar-sobre todo de nivel secundario-y de una manera didáctica y motivadora, se logra interiorizar en ellos los conceptos necesarios, que les permitirá tomar conciencia a la hora de ejercer acciones tributarias requeridas. Este es un proceso educativo que impregna en la persona-dentro de sus deberes y características personales - la responsabilidad de contribuir voluntariamente con los impuestos al país.

Desde otro orden de cosas, en el contexto peruano donde aún existe una presión insana que ahuyenta al mercado formal, también existe registro de algunas alternativas y acciones para impulsar la educación tributaria a mediano y largo plazo. Esto lo revela un informe de julio 2006, cuando se firmó un convenio de cooperación interinstitucional entre SUNAT y el Ministerio de Educación. Sin embargo, este parece haber sido obviado por ambas instituciones tal como lo describió Alcos (2016) al encontrar muy pocas temáticas sobre tributación en el currículo educativo nacional.

Quizás en este ámbito, es necesario tomar en cuenta lo que Comex Perú (2019) menciona al referir que el Perú enfrenta un alto nivel de informalidad, manteniendo en los últimos treinta años una tasa del 75\%, similar a países como los de África Subsahariana. No obstante, en el caso de Kenia se ha logrado evidenciar que este país ha sabido implementar la temática tributaria dentro del currículo del sistema educativo en materias como inglés, ciencias naturales y matemáticas. Por tanto, se busca introducir estos conceptos para que resulten naturales en los estudiantes durante su formación académica y profesional (Zambrano, 2019).

De igual manera, tal como lo sostiene la International and Ibero-American Foundation for Administration and Public Policies (FIIAPP), se requiere de alternativas de complemento y refuerzo a las técnicas ya conocidas, que fomenten la toma de conciencia acerca de la importancia de recaudar impuestos como alternativa a la presión tributaria; esto generaría el incremento de los ingresos por este concepto, los cuales son necesarios para financiar los 
servicios básicos que los ciudadanos necesitan. Por lo tanto, la educación en cultura tributaria no solamente debe ser una estrategia para recaudar más ingresos, sino deberá convertirse en un impulsor de cambio en la actitud del ciudadano. En este sentido, se podría empezar con los estudiantes de educación básica, hasta alcanzar los estudios superiores, a modo de transición natural hacia su incorporación al mercado laboral (FIIAPP, 2015).

En cuanto a las limitaciones de la presente investigación, los autores reconocen que la cantidad de participantes no permite una generalización de los resultados aplicable a poblaciones de adolescentes de otras regiones. Por ello, surge la necesidad de replicar el estudio en otros contextos del territorio nacional, ampliando el tamaño de población, además de mediciones a nivel longitudinal para determinar el impacto de este tipo de estrategias educativas provoca en los aprendizajes a corto y largo plazo.

\section{Conclusión}

El programa "¿Qué importante es tributar!" es eficaz para desarrollar un adecuado dominio de conocimientos, mejorar la percepción e incrementar el nivel de actitudes favorables hacia la tributación, en una muestra de adolescentes de la ciudad de Juliaca. El impacto positivo del programa en los participantes, evidencia la necesidad que el estado peruano pueda tomar en cuenta y aplicar este tipo de estrategias desde el ámbito escolar.

\section{Referencias}

Alcos, R. (2016). Cultura tributaria en el nivel de educación básica regular de la región Puno. (Tesis de Maestria). Recuperado de http://repositorio.unap.edu.pe/handle/UNAP/483?show=full

Camarero, L., Del Pino, J. A., y Mañas, B. (2015). Evolución de la cultura tributaria, coyuntura económica y expectativas vitales. Un estudio longitudinal. Madrid: Centro de Investigaciones Sociológicas.

Comex Perú. (2019). Los puntos claves para reducir la informalidad en el Perú, según ComexPerú. Recuperado de https://gestion.pe/economia/puntos-claves-reducir-nivelinformalidad-peru-nndc-265965?fbclid=IwAR1tA2A8eQ7iNYj0VU7-

Estrada, L. (2018). La Gallina de los huevos de oro. Recuperado de https://peru21.pe/opinion/gallina-huevos-oro-414586

FIIAAP (2015). Fomentando la cultura tributaria, el cumplimiento fiscal y la ciudadanía: Guía sobre educación tributaria en el mundo. Paris: OECD Publishing. doi: 10.1787/9789264222786-es 
Gómez, J., y Moran, D. (2016). La situación tributaria en America Latina: raices y hechos estilizados. Cuadernos de Economia, 35(17), 1-36.

INEI (2018). Indicadores Económicos. Recuperado de https://www.inei.gob.pe/media/MenuRecursivo/boletines/boletin-quincenal-14.pdf

Lahura, E. (2016). Sistema financiero, informalidad y evasión tributaria en el Perú. Banco Central de Reserva del Perú. Recuperado de http://www.bcrp.gob.pe/docs/Publicaciones/Documentos-deTrabajo/2016/documento-de-trabajo-11-2016.pdf

López, R. (2014). La evasión tributaria y su consecuencia en el desarrollo economico del pais. In Crescendo, 5(2), 253-266. Recuperado de https://revistas.uladech.edu.pe/index.php/increscendo/article/view/203

Mostacero, M., y Vasquez, K. (2014). Análisis comparativo de la aplicación de un programa de orientación y cultura tributaria en estudiantes del quinto año de secundaria de instituciones educativas públicas y privadas de la ciudad de Trujillo. (Tesis de licenciatura). Recuperado de http://repositorio.upao.edu.pe/bitstream/upaorep/710/1/MOSTACERO_MARIA_AN \%C3\%81LISIS_CULTURA_TRIBUTARIA.pdf

Nass, L. \& Merino, J. (2008). El experimento natural como un nuevo diseño cuasiexperimental en investigación social y de salud. Ciencia y Enfermeria, 14(2), 9-12. Recuperado de: https://scielo.conicyt.cl/pdf/cienf/v14n2/art02.pdf

Oliva, C. (2018). MEF: "La gran apuesta es la reducción de la evasión tributaria”. Recuperado de https://gestion.pe/economia/mef-gran-apuesta-reduccion-evasiontributaria-253461

Ruiz, J. (2017). La cultura tributaria y la gestión municipal. Quipukamayoc, 25(48), 49-60. Recuperado de https://revistasinvestigacion.unmsm.edu.pe/index.php/quipu/article/view/13992

Sanchez, A., Sartori, B., \& Salluca, J. (2017). La educación Tributaria a nivel secundaria y el impulso de la cultura tributaria en la región callao, año 2017. (Tesis de licenciatura). Recuperado de http://repositorio.unac.edu.pe/handle/UNAC/2286

SUNAT (2019). Cultura Tributaria y Aduanera. Recuperado de http://cultura.sunat.gob.pe/

Valencia, A. (2013). Neuro Tributos: ¿cómo internalizar la cultura tributaria para la gestión exitosa de los tributos?. Quipukamayoc, 21(40), 133-141. Recuperado de https://revistasinvestigacion.unmsm.edu.pe/index.php/quipu/article/view/6319

Vera, S. (2017). Motivación y cultura tributaria en estudiantes de primer ciclo, 2016. (Tesis de licenciatura). Recuperado de: http://repositorio.ucv.edu.pe/bitstream/handle/UCV/7747/Vera_LSP.pdf?sequen 
Zamora, Y. (2018). La evasión tributaria y su incidencia en la economía del Ecuador, 20102014. Quipucamayoc, 26(50), 21-29. Recuperado del 15 de mayo de: http://revistasinvestigacion.unmsm.edu.pe/index.php/quipu/article/view/14721

Zambrano, R. (2019). Centro interamericano de administraciones tributarias. Recuperado el 19 de Abril de 2019, de https://www.ciat.org/educacion-tributaria-o-tributacion-enla-educacion-un-acercamiento-diferente/ 